\title{
Further tests of the IRI and DGR model ionospheres
}

\author{
Žarko J. Kecić $\left({ }^{1}\right)$, Peter A. Bradley $\left({ }^{2}\right)$, Ljiljana R. Cander $\left({ }^{2}\right)$, Mike I. Dick $\left({ }^{2}\right)$ and Bruno Zolesi $\left({ }^{3}\right)$ \\ $\left.{ }^{1}\right)$ Geomagnetic Institute, Grocka, Belgrade, Yugoslavia \\ $\left({ }^{2}\right)$ Rutherford Appleton Laboratory, Chilton, Didcot, Oxon, U.K. \\ $\left(^{3}\right)$ Istituto Nazionale di Geofisica, Roma, Italy
}

\begin{abstract}
The present study discusses and compares the IRI and DGR ionospheric models using $N(h)$ profiles obtained during a high solar activity period at South Uist ionosonde station. After inversion, $N(h)$ profiles obtained from ionograms at South Uist have been compared with those derived from the IRI-90 and DGR ionospheric models. A small data set has been selected such that both geomagnetically quiet and disturbed conditions are represented. The importance of further comparisons with additional sets of $N(h)$ profiles under different solar-geophysical conditions and at more stations has been recognized.
\end{abstract}

Key words ionosphere - electron density models height profiles - testing - model comparison

\section{Introduction}

The last four decades have seen significant developments in electron-density height profile description. The most widely used empirical ionospheric model in science, engineering and education at present is a multi-segment model in terms of standard ionospheric characteristics known as the International Reference Ionosphere IRI (Bilitza et al., 1993). As the standard IRI (designated as IRI-90 in its current form) depends on the monthly CCIR «numerical maps» of $f_{0} F 2$ and $M(3000) F 2$ (CCIR Report 340, 1990), it describes monthly median profiles. Since the IRI profiles are critically dependent upon the adopted values of these ionospheric characteristics for many applications it is advantageous to use observed values of $f_{0} F 2$ and $M(3000) F 2$ wherever possible. On the other hand, Di Giovanni and Radicella (1990) introduced a regional, analytical model of the electron-density profile based on routine ionogram scaling - the DGR model.
Once models are developed, they should be tested before widespread use. In the work done to date, the focus has been almost exclusively on comparisons with monthly medians of observations. It would be possible to make comparisons with composite profiles derived by combining daily measurements within a month but recognising the uncertainties associated with limited daily sampling, here the emphasis is on tests using the individual measured profiles normalised to the peak densities and heights to assess in the light of day-to-day changes how well the models apply on particular days. With that in mind, we are testing the IRI-90 (Bilitza, 1990) and DGR (Di Giovanni et al., 1992) models with U.K. daily-hourly ionosonde data. This paper discusses these comparisons and offers some explanations of the results.

\section{Principal features of the IRI-90 and DGR models}

The structure of the IRI electron-density profile is shown in fig. 1. Although a fully 


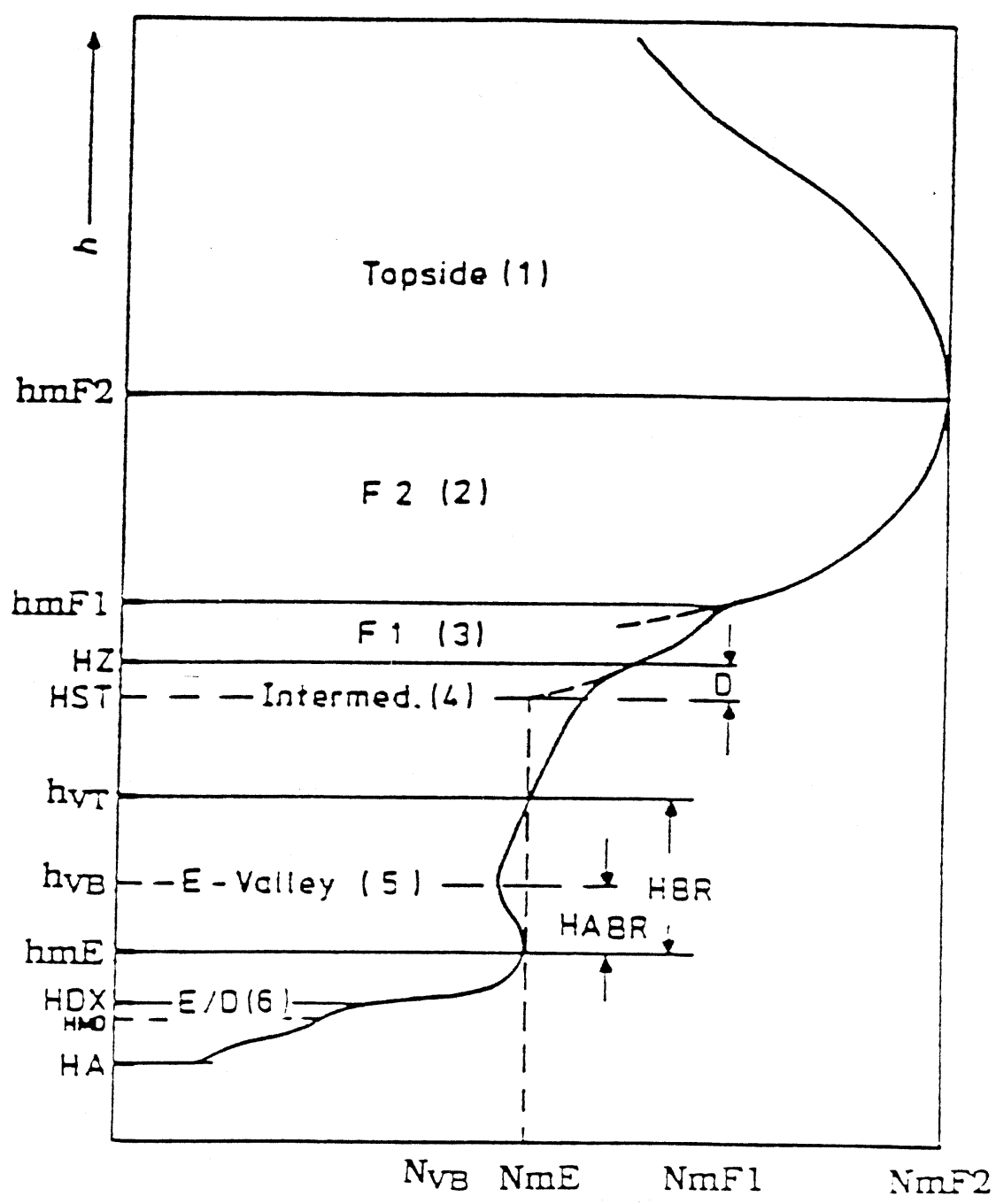

$\log N$

Fig. 1. Build-up of IRI electron-density profile.

analytic description is now also available in terms of LAY functions (Rawer, 1988), the most often used option is a description in «layered» form, meaning that separate mathematical expressions are formulated for the different height ranges, with parameters as follows: 1) peak densities: $N m E$, $N m F 1, N m F 2$; 2) peak heights: $h m E, h m F 1$, $h m F 2$; 3) layer thicknesses: $B_{0}, C_{1}$; 4) valley parameters: $h_{V T}, h_{V B}, N_{V B}$. In table I only the most important characteristics of the IRI-90 are included. Details of the functional description and explanations of the formulas adopted can be found in the IRI-90 guide book (Bilitza, 1990), and will not be repeated here. Finally, it should be empha- 
Table I. IRI-90.

F2-topside ( $\mathrm{hmF} 2$ to $1000 \mathrm{~km}$ )

$N m F 2$ - from CCIR or URSI coefficients;

hmF2 - from Bilitza et al. (1979);

profile shape - Booker function depending on geomagnetic latitude and $N m F$ (from Llewellyn and Bent, 1973).

\section{F2-bottomside (hmF1 or HZ to hmF2)}

Profile shape - from Ramakrishnan and Rawer (1972) obtained after smoothing the set of Llewellyn and Bent (1973), with thickness parameters $B_{1}=3$ and $B_{0}$ tabular with season, local time, solar epoch and latitude or from Gulyaeva (1987).

\section{F1-layer (hmF1 to HZ)}

$f_{0} F 1$ - Ducharme, Petrie and Eyfrig $(1971,1973)$;

$h m F 1-F 2$ bottomside intersection;

profile shape - parabolic, smoothly added to $F 2$ profile when present with the $F 1$-layer thickness $C_{1}$ based on ionosonde data.

\section{Intermediate region ( $H Z$ to $\left.h_{V T}\right)$}

Profile shape - parabolic merge between $F 2$-normalised segments and $E$-normalised segments (extends to $h m E$ if no valley).

E-peak and valley $\left(h_{V T}\right.$ to $\left.h m E\right)$

$f_{0} E$ - Kouris and Muggleton (1973) with Rawer and Bilitza (1990) twilight and night;

$h m E-105 \mathrm{~km}$;

profile shape - fifth-order polynomial with five valley parameters.

D-region and E-bottomside ( $h m E$ to $H A$ )

$\mathrm{Nm}$ and $h m D$ - Mechtley and Bilitza (1974) rocket data;

profile shape - third-order polynomial plus exponential link to $f_{0} E$.

sised that in the IRI-90 the experimental date near the $F 2$ peak were used as inputs in the model calculations.

Three basic criteria that should be taken into account to judge the success of a model have been described by Dudeney and Kressman (1986). They are: 1) how well the model matches the range of observed profiles; 2) the ease of obtaining the external data required to specify the profile, and 3 ) the simplicity of the mathematical expressions used to derive the profile. Di Giovanni and Radicella introduced an analytical model of the electron-density profile based on routinely scaled ionogram data which ap- pears to fulfil these criteria. The profile is described by one to three «Epstein layers» (Rawer, 1988) for the $F 2, F 1$ and $E$ layers of the ionosphere. The current internationally publicised version tested herewith (Radicella and Di Giovanni, 1991; Di Giovanni et $a l ., 1992)$ follows the equations in table II. The results obtained by a new version of the DGR model with revised $E / F 1$ valley by introducing the new value in the model parameter $B_{E}$ and modified topside by using simultaneous ionosonde and TEC data for the topside parameter $k$ (Di Giovanni et al., 1992) appear to be promising but require separate assessment. 
Table II. DGR.

$$
N(h)=\frac{4 N m * F 2 \exp (h-h m F 2) / B 2}{(1+\exp (h-h m F 2) / B 2)^{2}}+\frac{4 N m * F 1 \exp (h-h m F 1) / B 1}{(1+\exp (h-h m F 1) / B 1)^{2}}+\frac{4 N m * E \exp (h-h m E) / B e}{(1+\exp (h-h m E) / B e)^{2}}
$$

or

$$
N(h)=N F 2(h)+N F 1(h)+N E(h) .
$$

Here

$$
\begin{gathered}
N m * F 2=N m F 2-0.1 N m F 1, \\
N m * F 1=N m F 1-N F 2(h m F 1), \\
N m * E=N m E-N F 1(h m E=120 \mathrm{~km})-N F 2(h m E=120 \mathrm{~km}), \\
h m F 1=108.8+0.14 N m F 1\left[10^{9} / \mathrm{m}^{3}\right]+0.71\left|\operatorname{Dip}\left(^{\circ}\right)\right|
\end{gathered}
$$

$$
\begin{gathered}
h m F 2=\frac{1470 M F}{M+\Delta M}-176, M F=M \sqrt{\frac{0.0196 M^{2}+1}{1.2967 M^{2}-1}}, \Delta M=\frac{0.253}{f_{0} F 2 / f_{0} E-1.215}-0.012, \\
B 1=-8.52+8.3 N m F 1+0.12 h m F 1+15.6 \sin \left(\frac{2 \pi T+30}{356}+\pi / 2\right), \\
B 2(h \leq h m F 2)=\frac{0.385 N m F 2}{(d N / d h) \max }, B 2(h>h m F 2)=k B 2(h \leq h m F 2),
\end{gathered}
$$

where

$$
\begin{gathered}
\ln \left((d N / d h) \max \left[10^{9} \mathrm{~m}^{-3} \mathrm{~km}^{-1}\right]\right)=-3.467+0.857 \ln \left(f_{0} F 2[\mathrm{MHz}]\right)^{2}+2.02 \ln (M(3000) F 2), \\
k=-7.77+0.097(h m F 2 / B 2)^{2}+0.153 N m F 2\left[10^{11} / \mathrm{m}^{3}\right]
\end{gathered}
$$

(October to March),

$$
k=6.705-0.014 R 12-0.008 h m F 2
$$

(April to September),

with: $\quad T=$ day of the year; $M=M(3000) F 2 ; h m E=120 \mathrm{~km}$ and $B e=0.5 B_{F 1 \text { top }}$.

\section{Data base}

After inversion by the POLAN algorithm (Titheridge, 1985) which is the internationally adopted procedure, ionograms from South Uist ionosonde station $\left(57^{\circ} \mathrm{N}, 7^{\circ} \mathrm{W}\right)$ provide the electron-density profiles up to the peak of the $F 2$ layer. Above the peak Titheridge proposes a Chapman-like extrapolation which is a model shape not based on measured densities. It must also be noted that POLAN applies a somewhat arbitrarily chosen standard valley rather than one ob- tained separately from specific measurement for each occasion.

Data recorded in 1988 during the months of February (winter), March/April (spring) and May (summer) were used in the analysis. The February and May data represent a geomagnetically quiet period, April data are for a geomagnetically disturbed period, and March data cover both geomagnetically quiet and disturbed periods. Table III lists the days of ionosonde data used for testing the IRI-90 and DGR profiles in the next section. Typically there were some 6 hours of 
Table III. List of days used for testing the IRI90 and DGR profiles.

\begin{tabular}{lcrc}
\hline \hline \multicolumn{1}{c}{ Period } & $R_{i}$ & $A_{p}$ & Sum $K_{p}$ \\
\hline February 1988 & 40 & 15 & \\
3 February ( $Q 3$ day) & 68 & 3 & $6-$ \\
March 1988 & 76.2 & 14 & \\
24 March ( $Q 6$ day) & 83 & 5 & $9+$ \\
30 March (D3 day) & 108 & 34 & $32+$ \\
April 1988 & 88 & 16 & \\
6 April (D2 day) & 62 & 48 & $37+$ \\
May 1988 & 60.1 & 12 & \\
28 May ( $Q 2$ day) & 70 & 3 & 6 \\
\hline
\end{tabular}

observations considered on each of the 5 days.

Geomagnetic and solar data in table III are from the monthly publication «SolarGeophysical Data» issued by World Data Center A for Solar-Terrestrial Physics, NOAA. The ten most quiet days are labelled $Q 1-Q 10$ with $Q 1$ most quiet and the five most disturbed days are labelled $D 1-D 5$ with $D 1$ most disturbed. $R_{i}$ is the daily sunspot number, $A_{p}$ is the geomagnetic activity index and Sum $K_{p}$ is the daily sum of the geomagnetic planetary 3 -h-range index $K_{p}$.

\section{Profile testing}

An examination of all comparisons shows that the best agreement between the IRI-90 model and the data was found for 24 March 1988 (fig. 2). In this and all other tests presented the measured $f_{0} F 2$ were used as inputs to both sets of model calculations. This can be related to the fact that 24 March was a geomagnetically quiet day at a moderate level of solar activity. However, the largest discrepancies between the IRI-90 and our data occurred for 28 May 1988 (fig. 3) which was a day with very similar solar-geophysical conditions. Recently, Reinisch et al.
(1992) have presented the first results of the VIM study (Validation of Ionospheric Models) and shown that there are significant seasonal differences in the model-measurement agreements. This may be the explanation for our findings.

Compared to the IRI-90, the DGR model is much less complex and calculations show that it is able to reproduce the main features of the diurnal and seasonal variations of electron-density profiles (figs. 4 and 5). However, the agreement between the DGR model and the data is generally rather poor during the daytime (particularly in the valley region) but a little better at night. This is most evident in the case of the daytime differences in the summer (fig. 5). Note, however, the uncertainty of the inversion in the valley region as indicated above (Gulyaeva et al., 1990).

\subsection{Middle ionosphere (F2-peak to E-peak)}

To illustrate the match of the IRI-90 and DGR with F2-layer thickness, table IV shows whether the IRI-90 and DGR subpeak electron-density profiles heights are lower $(L)$, close to $(G)$ or higher $(H)$ than from POLAN. It can be seen from table IV that there are no consistent trends for geomagnetically quiet days. In contrast, in almost all the geomagnetically disturbed cases (30 March and 6 April) the IRI-90 and DGR models give electron-density profiles which are higher than or close to those from POLAN.

During the daytime, ionograms often show a characteristic $F 1$ feature, clearly identified by a cusp-like trace structure similar to those for the $E$ - and F2-peaks. Inverted into electron-density profiles, the $F 1$ feature translates into a small gradient discontinuity. Quite frequently a valley can be observed in the region above the $E$-peak. During the night the valley is always present and at mid-latitudes it is also often there during the daytime. The DGR model valleys in figs. 4 and 5 differ significantly from the POLAN profiles. Because the POLAN 



Fig. 2. Comparison of electron-density profiles given by IRI-90 (solid curve) and POLAN (dotted curve) for 24 March 1988 at 06, 08, 10, 12, 16 and 20 UT. 

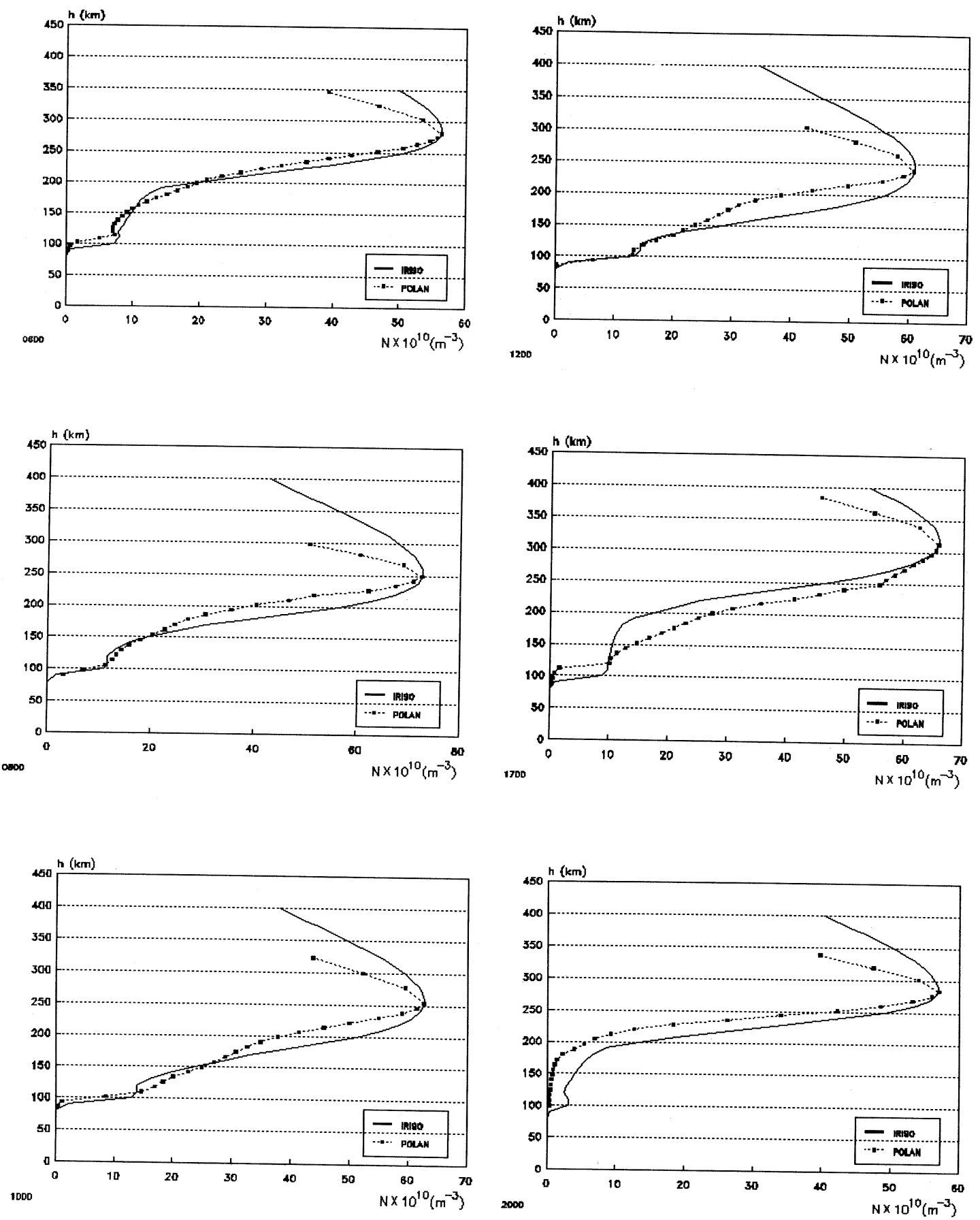

Fig. 3. Comparison of electron-density profiles given by IRI-90 (solid curve) and POLAN (dotted curve) for 28 May 1988 at 06, 08, 10, 12, 17 and 20 UT. 

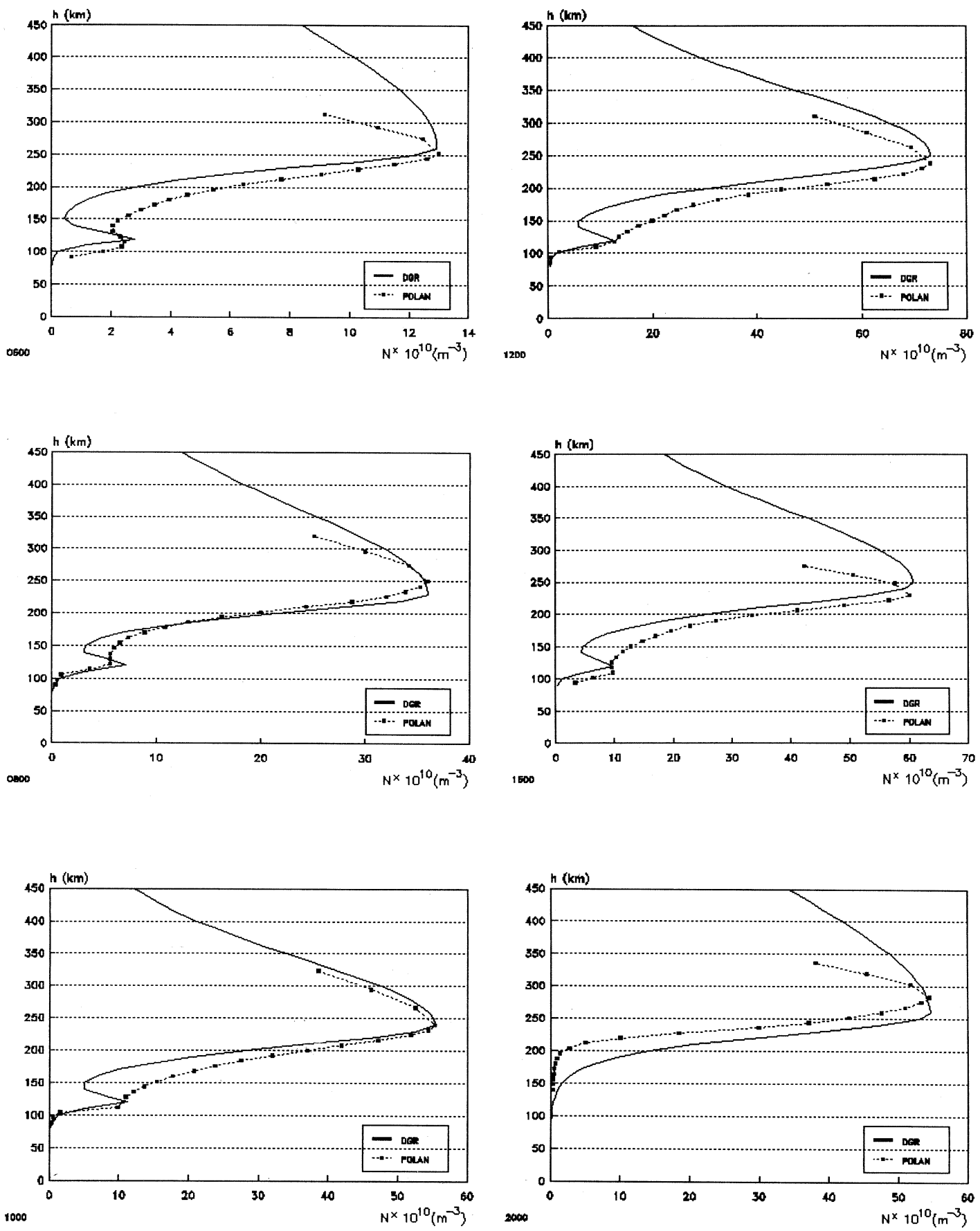

Fig. 4. Comparison of electron-density profiles given by DGR (solid curve) and POLAN (dotted curve) for 24 March 1988 at 06, 08, 10, 12, 16 and 20 UT. 

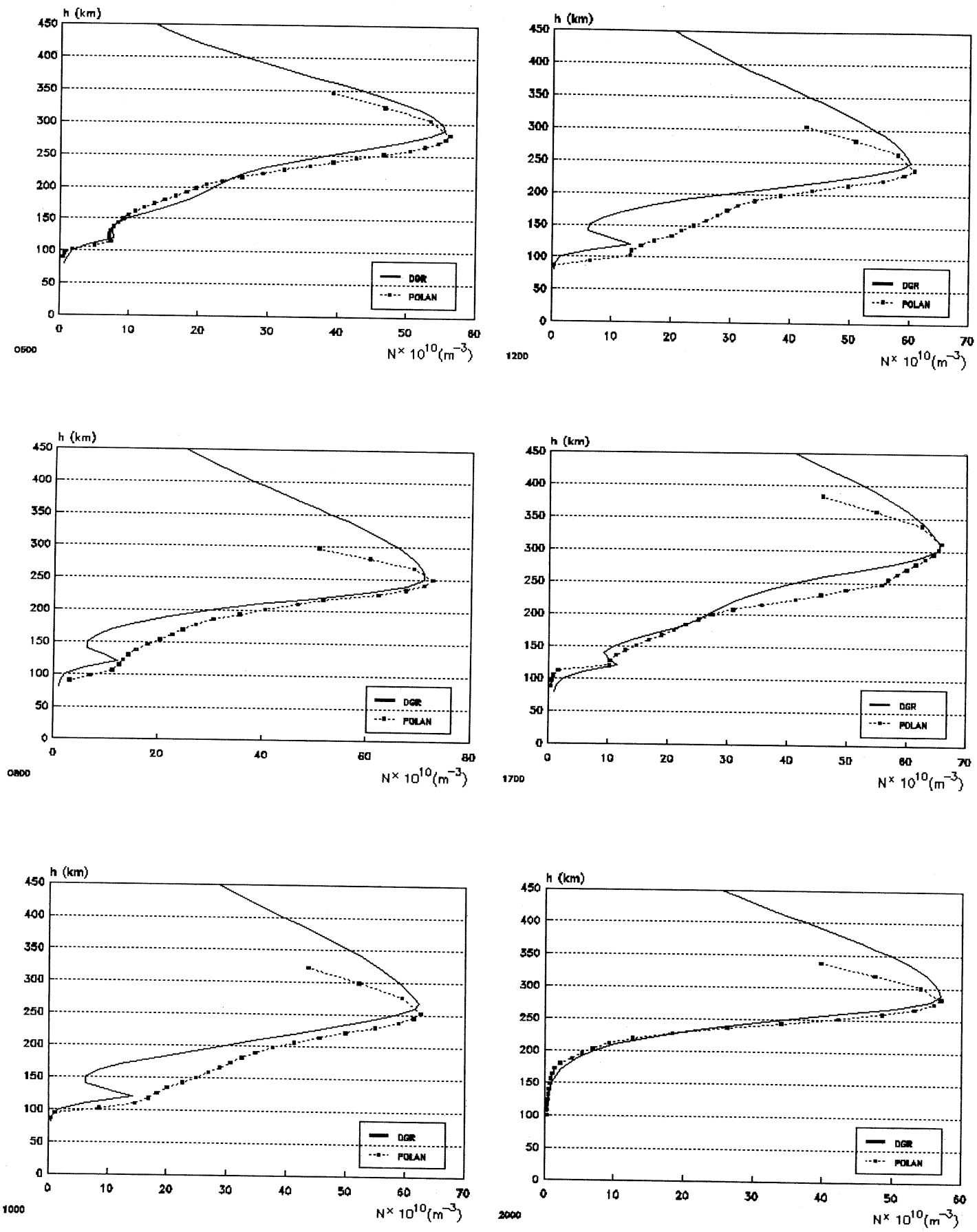

Fig. 5. Comparison of electron-density profiles given by DGR (solid curve) and POLAN (dotted curve) for 28 May 1988 at 06, 08, 10, 12, 17 and 20 UT. 
Table IV. Illustration of the match of IRI-90 and DGR thickness.

\begin{tabular}{ccccccccccccc}
\hline \hline Day/hour & \multicolumn{2}{c}{ 0600 UT } & \multicolumn{2}{c}{ 0800 UT } & \multicolumn{2}{c}{1000 UT } & \multicolumn{2}{c}{ 1200 UT } & \multicolumn{2}{c}{1600 UT } & \multicolumn{2}{c}{ 2000 UT } \\
\hline Models & IRI & DGR & IRI & DGR & IRI & DGR & IRI & DGR & IRI & DGR & IRI & DGR \\
\hline 3 February & H & H & H & H & L & G & G & L & L & G & H & G \\
24 March & G & H & G & G & G & H & L & H & L & H & G & L \\
30 March & H & H & G & G & H & H & H & H & H & H & H & H \\
6 April & H & H & H & G & G & H & G & L & H & H & H & H \\
28 May & L & G & L & H & L & H & L & H & L & H & L & G \\
\hline
\end{tabular}

gives a valley, it would be desirable to study the incoherent scatter significant variations of valley shapes by using the different true height algorithms.

\section{Data base and criteria to apply in testing model electron-density height profiles for adoption within PRIME}

The subject of model testing is one that has not been addressed systematically in the past and needs careful considerations. It is evident that so long as different groups use different data sets for the testing they are liable to arrive at inconsistent conclusions. So the need is for an agreed reference measured data set that can be compared with the different models. This matter is currently under active consideration within both the PRIME and URSI Ionospheric Informatics Working Groups with the hope that an unified approach and data set will emerge.

The comparison of measured and predicted profiles is not straight forward, even for individual cases where each exist. It could be argued that it all depends upon the application as to which part of the profile is the most important. For example, over a given oblique path densities near raypath apogee are more critical and these occur at heights which vary markedly with frequency and path length. Again, for some purposes such as assessing ionospheric effects on Earth-space links the topside ionosphere is of major importance. For $M F$ propagation and absorption assessments $E$-region densities are what matter most. However, whilst this means that sometimes one model may be best and sometimes another, we just cannot embody such a solution in our work and we must aim to adopt the general-purpose model that is best on average. So we need to generate a figure-of-merit that tests the profiles over all height ranges. This suggests to take the figure-of-merit as the RMS difference in the electron densities for a series of heights up to $1000 \mathrm{~km}$, the upper limit of our planned modelling, averaged over all the different profiles, probably with sample weighting in inverse proportion to the number of profiles for each occasion and location. An alternative figure-of-merit would be the root-mean-square or mean mdulus of the height differences between measured and model profiles at a selected set of electron densities. This would avoid problems with potentially unrealistic density differences at fixed heights under conditions of marked gradients, but would be sensitive to the selection of the density set for which to make the comparisons with changing relative $F 2, F 1$ and $E$ region densities. On balance the former approach is favoured.

At what heights then in turn shall the comparisons be made? The $E$-region is only $20 \mathrm{~km}$ thick, but the topside is maybe 700 $\mathrm{km}$ to our cut-off height. This suggests a varying separation depending on ionospheric region as follows: 


\begin{tabular}{|c|c|}
\hline $1000 \mathrm{~km}-h m F 2$ & every $100 \mathrm{~km}$ \\
\hline$h m F 2-H$ half $F 2$ & every $20 \mathrm{kr}$ \\
\hline$H$ half $F 2-h m E+10 \mathrm{~km}$ & every $10 \mathrm{k}$ \\
\hline$m E+10 \mathrm{~km}$ & \\
\hline
\end{tabular}

Now $F 2$ peak densities are perhaps a hundred times greater than those occurring in the $E$-region, so this means instead of working in absolute values at each height we should use relative figures expressed as a fraction of the measured values (or work with densities expressed in logarithmic units as in IRI). Where topside measurement data from incoherent-scatter stations or from topside-sounder satellites are not available, then of course the figure-of-merit must be derived for and relate only to the bottomside.

A further consideration is whether to normalise in any way the individual measured and predicted profiles before they are compared. For example, the IRI allows the possibility of being composed for a measured $f_{0} F 2$ and an $h m F 2$ given from a measured $M(3000) F 2$. On the one hand we do not want our figure-of-merit to be biased by errors in the peak density specification - we want it to quantify the goodness of the profile shape. But what do we do about all the other measured characteristics? Do we also insist on using the measured $f_{0} E$ ? Do we use a measured $f_{0} F 1$ with some profiles and not others? If we anchor all the profiles at too many places, they will then differ only in minor regards. It is our proposal that for comparison tests we anchor the separate profiles to the same $f_{0} F 2$ and $h m F 2$ given from the measurements, but that we do not attempt to anchor elsewhere.

\section{Conclusions}

The electron-density profiles derived from ionograms at South Uist were compared with those by the IRI-90 and DGR models using a small data set selected in such a way that both geomagnetically quiet and disturbed conditions are represented. Although the above models were not constructed to cover disturbed conditions, the IRI or DGR models could reproduce the profiles found after the SSC-disturbances. Our study also shows that while the models generally reproduce the overall diurnal and seasonal variations, an expansion of this study would be to 1) cover more ionospheric stations, and 2) test larger sets of the electron-density profiles under different solargeophysical conditions, such as would be the case using an extended VIM data base as discussed in the text.

\section{Acknowledgements}

The authors would like to thank very much Mrs. A. Vernon for her assistance in preparation of this paper. Dr. Lj.R. Cander would like to also thank the Rutherford Appleton Laboratory for giving her the opportunity to spend her sabbatical there. True height profiles used in this analysis were provided by Mr. T. Kelly, KEL Aerospace, Australia.

\section{REFERENCES}

Bilitza, D., W.M. SheikH and R. Eyfrig (1979): A global model for the height of the $F 2$-peak using $M(3000)$ values from the CCIR, Telecommun. J., 46, 549-553.

BilitzA, D. (1990): International Reference Ionosphere, NSSDC 90-22, (World Data Center A Rockets and Satellites, Greenbelt, U.S.A.).

Bilitza, D., K. Rawer, L. Bossy and T. Gulyaeva (1993): International Reference Ionosphere - past, present, and future: I. Electron density, Adv. Space Res., 13 (3), 3-13.

CCIR Report 340 (1990): CCIR atlas of ionospheric characteristics, ITU, Geneva.

Di GiovanNi, G. and S.M. Radicella (1990): An analytical model of the electron-density profile in the ionosphere, Adv. Space Res., 10 (11), 27-30.

Di Giovanni, G., S.M. RAdicella and M-L. Zhang (1992): Further development of the DGR ionospheric model and comparison with the IRI current model, COST238TD(92)021, May 1992.

Ducharme, E.D, L.E. Petrie and R. Eyfrig (1971, 1973): A method for predicting the $F 1$-layer critical 
frequency, Radio Sci., 6, 369-378 and Radio Sci., 8, 837-839.

DUDENEY, J.R. and R. I. KRESSMAN (1986): Empirical models of the electron concentration of the ionosphere and their value for radio communication purposes, Radio Sci., 21, 319-330.

GulyaEva, T.L. (1987): Progress in ionospheric informatics based on electron-density profile analysis of ionograms, Adv. Space Res., 7 (6), 39-48.

Gulyaeva, T.L., J.E. Titheridge and K. Rawer (1990): Discussion of the valley problem in $N(h)$ analysis of ionograms, $A d v$. Space Res., $10(8), 123-$ 126.

Kouris, S.S. and L.M. MugGleton (1973): Diurnal variation in the E-layer ionization, J. Atmos. Terr. Phys., 35, 133-139.

Llewellyn, S.K. and R.B. Bent (1973): Documentation and description on the Bent ionospheric model, AFCRL-TR-73-0657, Los Angeles, U.S.A.

Mechtley, E.A. and D. Bilitza (1974): Rep. IPWWB1, Fraunhofer-Inst f. physikal. Weltraumforschung, Freiburg i. Br., Germany.

Radicella, S.M. and G. Di Giovanni (1991): Im- provements of the Di Giovanni-Radicella model of electron-density profiles, presented at Workshop of IRI, Athens, Greece, COST238TD(92)008, 31 October 1991.

RAMAKRISHNAN, S. and K. RAWER (1972): Model electron-density profiles obtained by empirical procedures, Space Res., XII (Akademie-Verlag, Berlin), 1253-1259.

RAWER, K. (1988): Synthesis of ionospheric electrondensity profiles with Epstein functions, $A d v$. Space Res., 8 (4), 191-199.

RAWER, K. and D. BilitzA (1990): International Reference Ionosphere - plasma densities: status 1988, Adv. Space Res., 10 (8), 5-14.

Reinisch, B.M., D. Anderson, R.R. GemaChe, X. HuAng, C.F. Chen and D.T. DeCKer (1992): Validating ionospheric models with measured electron density profiles, Memoria, 16, (Publicaciones del Observatorio del Ebro, Roquetes), 41-52.

TITHERIDGE, J.E. (1985): Ionogram analysis with the generalized program POLAN, Report UAG-93 (World Data Center A for STP, NOAA, Boulder, U.S.A.). 